\title{
SENTINEL LYMPH NODE BIOPSY AFTER NEOADJUVANT CHEMOTHERAPY IN CLINICALLY NODE POSITIVE AXILLA OF BREAST CANCER
}

\author{
Osama Ali El Atrash, Mohamed El Sayed El Shinawi, Ahmed Gamal-Eldin \\ Osman and Ahmed Ragab Ibrahim Soliman
}

\begin{abstract}
:
Department of General Surgery, Faculty of Medicine - Ain Shams University, Cairo - Egypt.

Corresponding Author: Ahmed Ragab Ibrahim Mobile +2001014392197

E-mail:

a.ragab73@yahoo.com

Received: $25 / 6 / 2020$

Accepted: 21/7/2020

Online ISSN: 2735-3540

Background: In the modern treatment of breast cancer, the Sentinel Lymph node biopsy indication is present in many clinical circumstances, instead of classical axillary lymphadenectomy. It presents the advantage of conservative surgery, which significantly decreases the rate of postoperative complications, offering the patients a better quality of Life and reducing the costs of patients care after surgery. Sentinel Lymph node biopsy (SLNB) vs. axillary Lymph node dissection (ALND) in the current surgical treatment of early stage breast cancer.

Aim of the Work: The aim of the present study is to evaluate staging the axilla with sentinel lymph node biopsy after neoadjuvant chemotherapy in previous clinically node positive axilla in breast cancer to avoid the morbidity of an ALND.

Patients and Methods: This was a prospective cohort study was held in El Demerdash hospital Ain Shams University hospitals, Cairo, Egypt started with one hundred and twenty female patients with clinically node positive axilla in breast cancer. Approval of the Ethical Committee and written informed consent from all participants were obtained. Patient selection was achieved through a number of inclusion and exclusion criteria.

Results: Our study showed that SLNB is acceptable in cN1/2 patients who become cNO after neoadjuvant therapy: particularly in those with no residual disease in the breast, because SN status maintains its expected prognostic role, but also in cases with residual disease, because Axillary dissection (AD) has no influence on outcomes.

Conclusion: Sentinel lymph node biopsy will replace axillary lymph node dissection (ALND) in those patients with clinically node positive axilla without compromising their oncologic outcomes. Especially, rates of pathologic complete response ( $p C R)$ after neoadjuvant chemotherapy (NAC) have increased. In the neoadjuvant setting, SLN after NAC is feasible and accurate in clinically node positive patients as a continuous effort to avoid the morbidity of ALND.
\end{abstract}

Keywords: Sentinel lymph node biopsy, axillary lymph node dissection

\section{INTRODUCTION:}

Breast cancer is the most common invasive cancer in females worldwide. It accounts for $16 \%$ of all female cancers and
$22.9 \%$ of invasive cancers in women. $18.2 \%$ of all cancer deaths worldwide, including both males and females, are from breast cancer. ${ }^{(1)}$ 
Breast cancer is the most common cancer among Egyptian women, accounting for $35 \%$ of all cases treated at the National Cancer Institute. However, most cases are detected very late and, in many circumstances, are not treated. ${ }^{(2)}$

Axillary dissection is a surgical procedure that incises the axilla to identify, examine, or remove lymph nodes. Axillary dissection has been the standard technique used in the staging and treatment of the axilla in breast cancer. Axillary dissection should be reserved for patients with proven axillary disease preoperatively or with a positive sentinel node biopsy. ${ }^{(3)}$

Axillary dissection is only therapeutic in patients who are node positive. Therefore, performing axillary dissection in all patients would lead to an overtreatment of at least $60 \%$ of patients who are all node negative. The introduction of sentinel-node biopsy has changed the approach to the axilla, as the status of the axilla can be established with less morbidity for patients when compared with complete axillary dissection. There is an increasing use of neoadjuvant chemotherapy in breast cancer, and the optimal timing of sentinel node biopsy can be controversial. Axillary dissection is advocated for all patients who complete neoadjuvant treatment to down-stage the breast tumor, except when sentinel node biopsy is negative and undertaken prior to the neoadjuvant treatment. ${ }^{(3)}$

Neoadjuvant treatment is a widely accepted treatment for breast cancer and has been proved to be equally effective option when compared to adjuvant therapy. Advantages are determination of an individual tumor's chemosensitivity, reduce micro metastatic disease and decrease disease burden to allow less extensive surgery. ${ }^{(4)}$

In recent years, increasing rates of tumor down staging have been reported, rates that have approached 94\%, and more important, pCR is achieved by around 20_40\% of patients after NAT. Pathologic complete response has been associated with a better prognosis and overall survival. ${ }^{(5)}$

Sentinel node biopsy prior to chemotherapy could be considered the most accurate staging assessment of the axilla, while some would argue that performing the sentinel node biopsy after chemotherapy could allow patients who have had a complete pathological response to be spared the increased morbidity of an axillary clearance. ${ }^{(3)}$

Sentinel lymph node biopsy has replaced axillary lymph node dissection (ALND) in those patients with clinically node negative axilla and nowadays, patients with low burden disease in the SLNs may spare an ALND without compromising their oncologic outcomes. In the last decade, indications of neoadjuvant treatment (NAT) have been extended to patients with operable disease and with the use of targeted therapies; rates of pathologic complete response (pCR) after NAT have increased. In the neoadjuvant setting, SLN after NAT is feasible and accurate in clinically node negative patients and it has been explored in different randomized prospective studies in patients with clinically positive axilla in the continuous effort to avoid the morbidity of ALND. ${ }^{(6)}$

\section{AIM OF THE WORK:}

The aim of the present study is to evaluate staging the axilla with sentinel lymph node biopsy after neoadjuvant chemotherapy in previous clinically node positive axilla in breast cancer to avoid the morbidity of an ALND.

\section{PATIENTS AND METHODS:}

This was a prospective cohort study was held in El Demerdash hospital Ain Shams University hospitals, Cairo, Egypt started with one hundred and twenty female patients with clinically node positive axilla in breast cancer. Approval of the Ethical Committee 
and written informed consent from all participants were obtained. Patient selection was achieved through a number of inclusion and exclusion criteria.

Place and timing of the study: our study was held at El Demerdash hospital between April 2017 till December 2019.

Inclusion Criteria: Patients ranging age from 20 to 70 years old. Patients with positive axillary L.Ns confirmed with fine needle aspiration biopsy (FNAB) before neoadjuvant chemotherapy who converted to clinically \& radiologically negative axillary lymph nodes post neoadjuvant chemotherapy(NAC). Able to tolerate General Anesthesia

Exclusion criteria: Patients with any other malignancies. Patients with radiologically positive axillary lymph nodes post neoadjuvant chemotherapy (NAC). Patients with Lymphadenopathy due to any cause other than breast cancer. Patients with any organ metastasis secondary to breast cancer.

All patients included in the study were candidates for: Clinical assessment: Detailed medical and family history. Full Clinical examination including both breasts and axillae. Investigation: Routine laboratory investigations (CBC, coagulation profile, liver functions, kidney functions, RBS). ECG.

Postoperatively: Broad spectrum antibiotics. Surgical site suction drain was placed intraoperatively and was kept for 714 days. Hospital stay: from 24 to 48 hours. Follow up: after 1 week for result of histopathological examination of SLN, then 2 weeks to remove drain, then 6 months. This was carried out by clinical assessment.

\section{Statistical Analysis:}

Data were collected, revised, coded and entered to the Statistical Package for Social Science (IBM SPSS) version 23. The quantitative data were presented as mean,
Sonomammography on both breasts and axillae. Pelviabdominal CT and CT chest. Fine needle aspiration biopsy and cytology (FNABC) of radiologically positive axillary L.Ns. Chemotherapy: Each patient received 6 cycles of neoadjuvant chemotherapy. After completion of chemotherapy, we repeated breast ultrasound to ensure radiolagically negative axilla (complete radiological response) post NAC that achieved in 50 patients and hence included in our study but the other 70 patients did not achieve complete radiological response though they were excluded from our study. Preoperatively: Patients were fully informed about the Risks and Benefits of the Procedures. Informed Consent was obtained from every Patient. Intraoperatively: Patients underwent sentinel lymph node biopsy using $1 \mathrm{~cm}$ of patent blue dye injected retroareolar at 3,6,9,12 o'clock then applying massage for whole breast for 15-20 minutes to allow the dye to reach from retroareolar plexus of Sappi to sentinel lymph node at axilla. After removal of sentinel lymph node (number 3-4 lymph nodes), was sent for pathologist for both intraoperative frozen and postoperative paraffin histopathological examination. Then completion of formal axillary clearance to assess safety and accuracy of sentinel lymph node biopsy (SLNB).

standard deviations and ranges when parametric. Also qualitative variables were presented as number and percentages.

The comparison between groups regarding qualitative data were done by using Chi-square test and/or Fisher exact test only when the expected count in any cell found less than 5 .

The comparison between two independent groups with quantitative data and parametric distribution were done by using Independent t-test test. 


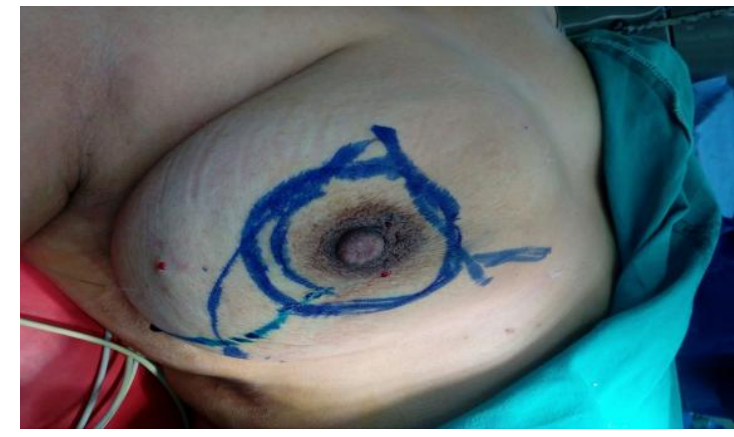

Figure (1): Retroareolar injection of patent blue dye.

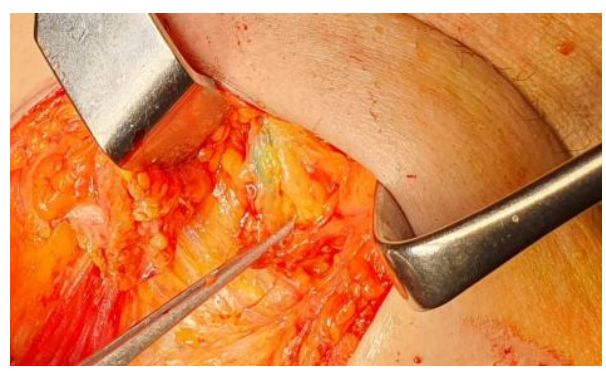

Figure (3): Shows patent blue dye stains sentinel lymph node

\section{Receiver operating characteristic curve} $(\boldsymbol{R O C})$ was used in the qualitative form to assess the diagnostic accuracy of paraffin section (axilla) and frozen section in differentiation between positive and negative cases taking paraffin section (SLN) as a gold standard.

The confidence interval was set to $95 \%$ and the margin of error accepted was set to $5 \%$. So, the p-value was considered significant as the following:

$\mathrm{P}>0.05$ : Non significant

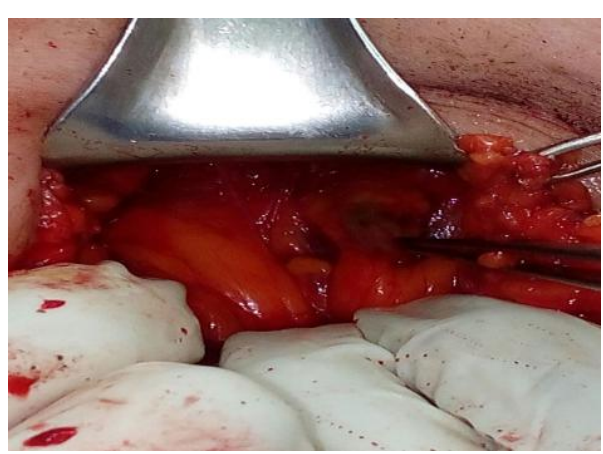

Figure (2): Shows patent blue dye stains sentinel lymph node

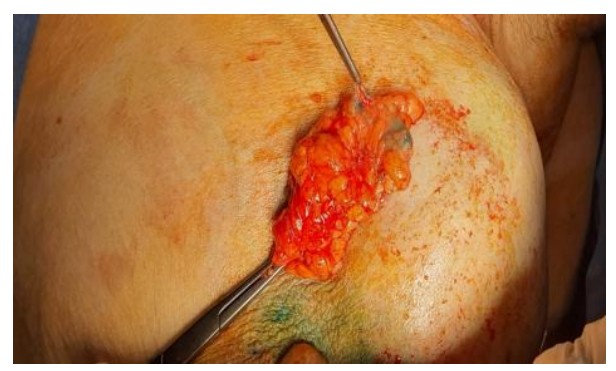

Figure (4): After excision of sentinel lymph node

$\mathrm{P}<0.05$ : Significant $\mathrm{P}<0.01$ : Highly significant.

\section{RESULTS:}

50 patients were included in our study, those are female patients with early breast cancer; without any other malignancies and without any Lymphadenopathy due to any cause other than breast cancer with mean Age 47.6 years.

Table (1): Shows demographic data about patient age, family history and side of tumor.

\begin{tabular}{|l|l|c|}
\hline \multicolumn{2}{|c|}{} & No.=50 \\
\hline \multirow{2}{*}{ Age (years) } & Mean \pm SD & $47.60 \pm 13.41$ \\
\cline { 2 - 3 } & Range & $22-68$ \\
\hline \multirow{2}{*}{ Family history } & Negative & $17(34.0 \%)$ \\
\cline { 2 - 3 } & Positive & $33(66.0 \%)$ \\
\hline \multirow{2}{*}{ Side of tumor } & Right & $31(62.0 \%)$ \\
\cline { 2 - 3 } & Left & $19(38.0 \%)$ \\
\hline
\end{tabular}


Table (2): Shows Site of tumor among the studied patients.

\begin{tabular}{|c|c|c|c|c|}
\hline \multirow{2}{*}{ Site } & \multicolumn{2}{|c|}{ Left } & \multicolumn{2}{c|}{ Right } \\
\cline { 2 - 5 } & No. & $\%$ & No. & $\%$ \\
\hline 1 & 3 & $15.8 \%$ & 0 & $0.0 \%$ \\
\hline 2 & 3 & $15.8 \%$ & 0 & $0.0 \%$ \\
\hline 3 & 4 & $21.1 \%$ & 2 & $6.5 \%$ \\
\hline 4 & 1 & $5.3 \%$ & 1 & $3.2 \%$ \\
\hline 5 & 1 & $5.3 \%$ & 1 & $3.2 \%$ \\
\hline 7 & 2 & $10.5 \%$ & 2 & $6.5 \%$ \\
\hline 8 & 1 & $5.3 \%$ & 1 & $3.2 \%$ \\
\hline 9 & 0 & $0.0 \%$ & 4 & $12.9 \%$ \\
\hline 10 & 2 & $10.5 \%$ & 8 & $25.8 \%$ \\
\hline 11 & 1 & $5.3 \%$ & 8 & $25.8 \%$ \\
\hline 12 & 1 & $5.3 \%$ & 4 & $12.9 \%$ \\
\hline
\end{tabular}

Table (3): Shows tumor parameters and patients weight

\begin{tabular}{|l|c|c|}
\hline \multicolumn{2}{|c|}{} & No. $=50$ \\
\hline \multirow{2}{*}{ Breast mass size $(\mathrm{cm})$} & Mean \pm SD & $3.23 \pm 0.64$ \\
\cline { 2 - 3 } & Range & $1.5-4.2$ \\
\hline \multirow{2}{*}{ BIRADS } & 4 & $32(64 \%)$ \\
\cline { 2 - 3 } & 5 & $18(36 \%)$ \\
\hline \multirow{2}{*}{ PT Weight } & Mean \pm SD & $77.84 \pm 8.22$ \\
\cline { 2 - 3 } & Range & $57-90$ \\
\hline
\end{tabular}

Table (4): Shows number of SLN

\begin{tabular}{|l|l|c|c|}
\hline \multicolumn{2}{|c|}{} & Frequency & Percent \\
\hline \multirow{2}{*}{ Number of SLN } & 3 & 28 & $56 \%$ \\
\cline { 2 - 4 } & 4 & 22 & $44 \%$ \\
\hline
\end{tabular}

Table (5): Shows results of frozen section, paraffin section SLN and axilla among the studied patients

\begin{tabular}{|l|l|c|}
\hline \multicolumn{2}{|c|}{} & No.=50 \\
\hline Frozen section & Negative & $35(70.0 \%)$ \\
\cline { 2 - 3 } & Positive & $15(30.0 \%)$ \\
\hline \multirow{2}{*}{ Paraffin section (SLN) } & Negative & $38(76.0 \%)$ \\
\cline { 2 - 3 } & Positive & $12(24.0 \%)$ \\
\hline Paraffin section (axilla) & Negative & $42(84.0 \%)$ \\
\cline { 2 - 3 } & Positive & $8(16.0 \%)$ \\
\hline
\end{tabular}

Table (6): Comparison between frozen section, paraffin section SLN and axilla of the studied patients.

\begin{tabular}{|l|c|c|c|c|c|c|}
\hline & $\begin{array}{c}\text { Frozen } \\
\text { section }\end{array}$ & $\begin{array}{c}\text { Paraffin } \\
\text { section (SLN) }\end{array}$ & $\begin{array}{c}\text { Paraffin } \\
\text { section (axilla) }\end{array}$ & $\begin{array}{c}\text { Test } \\
\text { value* }\end{array}$ & P-value & Sig. \\
\cline { 1 - 4 } Negative & $35(70.0 \%)$ & $38(76.0 \%)$ & $42(84.0 \%)$ & 2.758 & 0.252 & NS \\
\hline Positive & $15(30.0 \%)$ & $12(24.0 \%)$ & $8(16.0 \%)$ & & \\
\hline
\end{tabular}

$\mathrm{P}>0.05$ : Non significant (NS); P $<0.05$ : Significant (S); P 0.01 : Highly significant (HS)

*: Chi-square test 
Table (7): Shows all factors that affect positivity and negativity of paraffin section of SLN

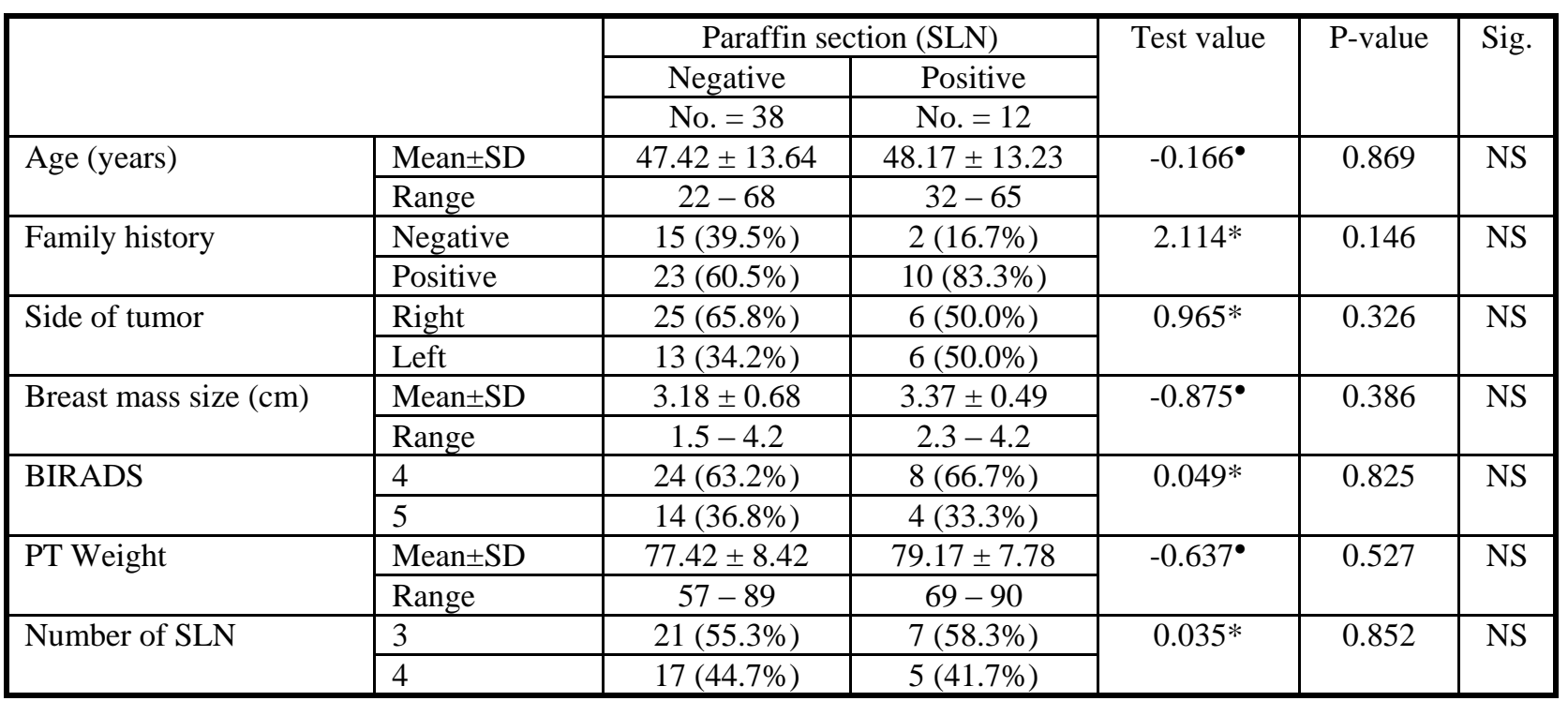

$\mathrm{P}>0.05$ : Non significant (NS); P < 0.05: Significant (S); P < 0.01: Highly significant (HS)

*: Chi-square test; $\bullet$ : Independent t-test

Table (8): Shows relation of paraffin section (SLN) with frozen section and paraffin section (axilla) among the studied patients

\begin{tabular}{|c|c|c|c|c|c|c|}
\hline & \multicolumn{2}{|c|}{ Paraffin section (SLN) } & \multirow[t]{3}{*}{ Test value } & \multirow[t]{3}{*}{ P-value } & \multirow[t]{3}{*}{ Sig. } \\
\hline & & Negative & Positive & & & \\
\hline & & No. $=38$ & No. $=12$ & & & \\
\hline \multirow[t]{2}{*}{ Frozen section } & Negative & $35(92.1 \%)$ & $0(0.0 \%)$ & \multirow[t]{2}{*}{36.842} & \multirow[t]{2}{*}{0.000} & \multirow[t]{2}{*}{ HS } \\
\hline & Positive & $3(7.9 \%)$ & $12(100.0 \%)$ & & & \\
\hline \multirow{2}{*}{$\begin{array}{l}\text { Paraffin section } \\
\text { (axilla) }\end{array}$} & Negative & $38(100.0 \%)$ & $4(33.3 \%)$ & \multirow[t]{2}{*}{30.159} & \multirow[t]{2}{*}{0.000} & \multirow[t]{2}{*}{ HS } \\
\hline & Positive & $0(0.0 \%)$ & $8(66.7 \%)$ & & & \\
\hline
\end{tabular}

P > 0.05: Non significant (NS); P < 0.05: Significant (S); P < 0.01: Highly significant (HS)

*: Chi-square test; $\bullet$ : Independent t-test

Completion of formal axillary clearance was done to assess safety and accuracy of sentinel lymph node biopsy (SLNB) revealed that there is no difference between results of positivity and negativity of both sentinel lymph nodes and other lymph nodes in axillary clearance.

Table (9): Shows diagnostic accuracy of frozen section and paraffin section (axilla) taking paraffin section (SLN) as a gold standard.

\begin{tabular}{|l|c|c|c|c|c|}
\hline & Sensitivity & Specificity & PPV & NPV & Accuracy \\
\hline Axilla & $100 \%$ & $66.7 \%$ & $100.0 \%$ & $90.5 \%$ & $92.0 \%$ \\
\hline Frozen section & $100.0 \%$ & $92.1 \%$ & $80.0 \%$ & $100.0 \%$ & $94.0 \%$ \\
\hline
\end{tabular}

Table (10): Shows biological classification of patients

\begin{tabular}{|l|c|c|}
\hline & Number of patients(50) & Percentage \\
\hline Luminal A & 8 & $16 \%$ \\
\hline Luminal B & 7 & $14 \%$ \\
\hline HER 2neu & 18 & $36 \%$ \\
\hline Triple -ve & 17 & $34 \%$ \\
\hline
\end{tabular}




\section{DISCUSSION:}

Breast cancer is the most common invasive cancer in females worldwide. It accounts for $16 \%$ of all female cancers and $22.9 \%$ of invasive cancers in women. $18.2 \%$ of all cancer deaths worldwide, including both males and females, are from breast cancer $^{(1)}$

SLNB after NAC can be used to avoid non-therapeutic ALND and its associated morbidity in the 30 to $40 \%$ of patients that present with positive LNs and are downstaged by NAC. As NAC (including the use of targeted agents) improves, the potential number of patients who could avoid ALND will certainly increase ${ }^{(7)}$

Our study started with 120 patients diagnosed breast cancer who are clinically and pathologically positive axilla. they all underwent 6 cycles of chemotherapy and then underwent breast ultrasound showed that 70 patients did not achieve complete radiological response (CRR) so they were excluded from our study. on the other hand, 50 patients achieved CRR and hence included in our study.

Those 50 patients were included in our study with mean age 47.6 years and their age ranges from 22-68 years old. While American Cancer Society revealed that mean age of patients of breast cancer was 50 years old ${ }^{(8)}$

In our study family history was positive in 33 patients with their percentage of $66 \%$. While American Cancer Society revealed that family history was positive in more than $70 \%$ of patients of breast cancer. ${ }^{(8)}$

This study showed that the site of tumor in 31 patients present with breast cancer on their right side with percent of $62 \%$ and the other 19 patients present with breast cancer on left side with their percentage of $38 \%$.

In our study the most common site of tumor is at $10 \& 11$ o'clock in right breast and at $2 \& 3$ o'clock in left breast and this is consistent with another study that revealed the most common site of tumor is upper outer quadrant at $10 \& 11$ o'clock in right breast and at $2 \& 3$ o'clock in left breast. ${ }^{(9)}$

This study showed that the size of mass ranges from $1.5 \mathrm{~cm}$ to $4.2 \mathrm{~cm}$ with mean size of $3.23 \mathrm{~cm}$ in diameter. It also showed that 32 patients were BIRADS 4 with percentage of $64 \%$ and the other 18 patients were BIRADS 5 with percentage of $36 \%$. Regarding patient weight, it ranges from 57$90 \mathrm{~kg}$ with mean weight of $77.84 \mathrm{~kg}$.

Then Patients underwent sentinel lymph node biopsy using $1 \mathrm{~cm}$ of patent blue dye injected retroareolar at 3,6,9,12 o'clock then applying massage for whole breast for 15-20 minutes to allow the dye to reach from retroareolar plexus of Sappi to sentinel lymph node at axilla.

After removal of sentinel lymph node (number 3-4 lymph nodes), sent for pathologist for both intraoperative frozen and postoperative paraffin histopathological examination.

In this study number of SLN, they were 3 lymph nodes in 28 patients with their percentage of $56 \%$ and were 4 lymph nodes in other 22 patients with percentage of $44 \%$.

The SLN is the sole tumor-bearing node in up to 60 percent of cases overall, and in almost 90 percent of patients who harbor only micrometastatic disease. These observations have led to speculation that completion ALND may not be necessary in selected patients with a positive SLNB in less than three nodes because the need for systemic therapy is established and the risk of an axillary recurrence appears to be low. (10)

In our study frozen section showed negative lymph nodes in 35 patients with percentage of $70 \%$ and positive lymph nodes in other 15 patients with percentage of $30 \%$ but paraffin section of SLN showed negative lymph nodes in 38 patients with percentage 
of $76 \%$ and positive lymph nodes in 12 patients with percentage of $24 \%$.

Other study showed that Data on frozen section assessment however is emerging showing sufficient, and perhaps superior intraoperative evaluation of nodal tumor burden, despite initial concerns that it consumes more of the specimen than cytological assessment. Analysis of published data to date shows that the accuracy of frozen section analysis with a combination of $\mathrm{H} \& \mathrm{E}$ staining and immunohistochemistry on sentinel lymph nodes lie between 73 to $96 \%$. ${ }^{(11)}$

Completion of formal axillary clearance was done to assess safety and accuracy of sentinel lymph node biopsy (SLNB) and sent for paraffin section and showed that negative lymph nodes in 38 patients with percentage of $76 \%$ and positive lymph nodes in 12 patients with percentage of $24 \%$ revealed that there is slight difference between results of positivity and negativity of both sentinel lymph nodes and other lymph nodes in axillary clearance.

Hormone receptor (HR) status is long known as independent predictor for chemotherapy response. In our study we found response to neoadjuvant chemotherapy is better in patients with positive HER 2 neu and in triple negative patients. We found 18 patients are positive for HER 2 neu with percentage of $36 \%$ and another 17 patients are triple negative with percentage of $34 \%$ and this consistent with other studies showed that the rate of pathological complete responses (pCR) differs between biological phenotypes, patients with a HR+/HER2tumor had a very low chance of achieving a pCR but had still an excellent prognosis. Whereas patients with HR-/HER2+ tumors have only an excellent prognosis when achieving a pCR. ${ }^{(12)}$

There are several factors affecting the success and accuracy of sentinel lymph node as using dual tracer with a radiolabelled colloid and a patent blue dye should be used; this will increase the SLN detection rate and the number of SLNs identified also decrease the FNR. ${ }^{(7)}$

Other limitations to our study are; the small group of patients, short follow up periods, use of patent blue dye only not using radiolabeled colloid.

The 2014 American Society of Clinical Oncology (ASCO) guidelines and 2015 National Comprehensive Cancer Network $(\mathrm{NCCN})$ guidelines reflect the most conservative treatment, recommending completion ALND only for patients with more than 2 positive lymph nodes while most women who meet precise criteria (T1-2 tumor, 1-2 positive lymph-nodes, absence of previous neoadjuvant therapy and for which is planned breast conserving surgery with whole breast irradiation) should not undergo ALND. ${ }^{(13)}$

Micrometastases at sentinel node biopsy should not be considered an indication to ALND anymore, although in some countries this practice is not yet adopted. In $2011 \mathrm{St}$ Gallen Consensus Conference has already moved forward a more conservative direction recommending that micrometastases in a single SLN should not be an indication for ALND irrespectively of the type of breast surgery given. The American Society of Clinical Oncology (ASCO) practice guidelines now allow to omit ALND to patients with micrometastatic and even 1-2 macrometastatic positive SLNs. ${ }^{(14)}$

The Venezuelan Mastology Society published its Consensus of Sentinel Lymph Node in Mammary Carcinoma in 2010. This recommends performing ALND in all cases of macrometastasis and leaves the decision in the hands of each working group in cases of micrometastasis.

The American Society of Clinical Oncology (ASCO) recommends performing 
ALND in all patients with SLN, whether they have macro or micrometastasis.

Eventually our study showed that SLNB is acceptable in cN1/2 patients who become $\mathrm{cN} 0$ after neoadjuvant therapy particularly in those with no residual disease in the breast, because SN status maintains its expected prognostic role, but also in cases with residual disease, because Axillary dissection (AD) has no influence on outcomes.

Today there is growing updates to increase the efficacy and efficiency of SLN though a new approach called Targeted Axillary Dissection (TAD) was introduced. It requires the marking of metastatic nodes before the neoadjuvant treatment. In cases with initially pathologic nodes they recommend marking of the most explicit metastatic node to enable TAD. The marked node is likely to reflect the status of the lymph nodes after neoadjuvant treatment more accurately than common sentinel nodes. ${ }^{(15)}$

Another study presents the first documented case of the use of magnetic seeds to mark axillary lymph node metastasis in breast cancer prior to neoadjuvant chemotherapy with magnetometer-guided intraoperative identification of the marked lymph node. The magnetic seed is a new technology that uses a $1 \times 5 \mathrm{~mm}$ surgical stainless steel marker. It can be detected using a magnetometer (Sentimag, Endomag, Cambridge, UK), which provides information on the direction to the marker and how far away it is. This means the marked node can be located in a similar fashion as the way in which a radioactive iodine seed would be detected, but without the regulatory problems associated with the use of radioactive materials. They believe marking positive lymph nodes before commencing neoadjuvant treatment with a magnetic seed could provide a simple and effective means of intraoperative localization of the marked node. ${ }^{(16)}$

\section{Conclusion:}

Sentinel lymph node biopsy will replace axillary lymph node dissection (ALND) in those patients with clinically node positive axilla without compromising their oncologic outcomes. Especially, rates of pathologic complete response (pCR) after neoadjuvant chemotherapy (NAC) have increased. In the neoadjuvant setting, SLN after NAC is feasible and accurate in clinically node positive patients as a continuous effort to avoid the morbidity of ALND.

\section{REFERENCES}

1. Nordqvist Christian, Breast cancer is a kind of cancer that develops from breast cells. http://www.medicalnewstoday.com/articles/37136 .php. (May, 2016)

2. Ibrahim Amal S, Hussein M Khaled, Nabiel Mikhail, Hossam Kamel, Cancer Incidence in Egypt, Journal of Cancer Epidemiology Volume 2014 ,Article ID 437971, 18 pages (September, 2014)

3. Singhal Hemant, Avi Agrawal, Erik D Schraga, Axillary Dissection. http://emedicine.medscape.com/article/1894 763-overview. (Nov, 2016)

4. Esserman LJ, Berry DA, DeMichele A, et al. Pathologic complete response predicts recurrence-free survival more effectively by cancer subset: results from the I-SPY 1 TRIALeCALGB 150007/150012, ACRIN 6657. J Clin Oncol 2012; 30(26):3242-9.

5. Shin HC, Han W, Moon H-G, et al. Breastconserving surgery after tumor downstaging by neoadjuvant chemotherapy is oncologically safe for stage III breast cancer patients. Ann Surg Oncol 2013; 20(8):25829.

6. Rubio I.T, Sentinel lymph node biopsy after neoadjuvant treatment in breast cancer: Am J surg, Dec; 176(6): 532-7 (November, 2015)

7. Kuehn T, Bauerfeind I, Fehm T, Fleige B, Hausschild M, Helms G and Schmatloch S (2013): Sentinel-lymph- node biopsy in patients with breast cancer before and after 
neoadjuvant chemotherapy (SENTINA): a prospective, multicentre cohort study. The lancet oncology; 14(7): 609-618.

8. DeSantis CE, Ma J, Jemal A. Trends in stage at diagnosis for young breast cancer patients in the United States. Breast Cancer Res Treat. 2019;173(3):743-747

9. Rummel S, Hueman MT, Costantino N, Shriver CD, Ellsworth RE. Tumour location within the breast: Does tumour site have prognostic ability? Ecancermedicalscience. 2015 Jul 13;9:552

10. Jeruss JS, Winchester DJ, Sener SF, et al. Axillary recurrence after sentinel node biopsy. Ann Surg Oncol. 2005;12:34.

11. Rahman M, Mohammed S. Breast cancer metastasis and the lymphatic system. Oncology letters. 2015; 10(3):1233-9.

12. Huober J, von Minckwitz G, Denkert C, Tesch H, Weiss E, Zahm DM, Belau A, Khandan F, Hauschild M, Thomssen C, Högel B. Effect of neoadjuvant anthracycline-taxane-based chemotherapy in different biological breast cancer phenotypes: overall results from the GeparTrio study. Breast cancer research and treatment. 2010 Nov 1;124(1):133-40.
13. Lyman GH, Temin S and Edge SB (2014): Sentinel lymph node biopsy for patients with early-stage breast cancer: American Society of Clinical Oncology clinical practice guideline update. J Clin Oncol. 2014; 32(13): 1365-83.

14. Esposito E, Sollazzo V, Di Micco R, Cervotti M, Luglio $G$, Benassai $G$ and Forestieri P (2016): Can axillary node dissection be safely omitted in the elderly? A retrospective study on axillary management of early breast cancer in older women. International Journal of Surgery; 33: S114-S118.

15. Coufal O, Zapletal O, Gabrielová L, Fabian $\mathrm{P}$, Schneiderová M.Targeted axillary dissection and sentinel lymph node biopsy in breast cancer patients after neoadjuvant chemotherapy - a retrospective study. Rozhl Chir. Winter 2018;97(12):551-557.

16. García-Moreno JL, Benjumeda-Gonzalez AM, Amerigo-Góngora M, Landra-Dulanto PJ, Gonzalez-Corena Y, Gomez-Menchero J (2019): Targeted axillary dissection in breast cancer by marking lymph node metastasis with a magnetic seed before starting neoadjuvant treatment. J Surg Case Rep. 2019(11): 344. 


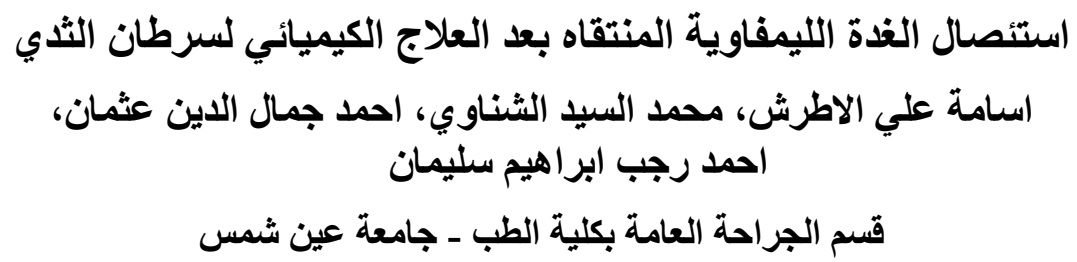

المقدمة: تشريح الإبط هو اجر اء علاجي في المرضى الذين لايهم عقدة إيجابية فقط. لذللك، أداء التشريح الإبطي في

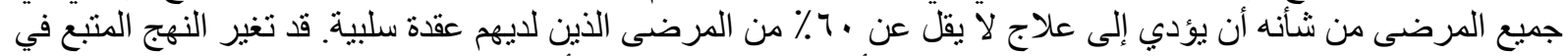

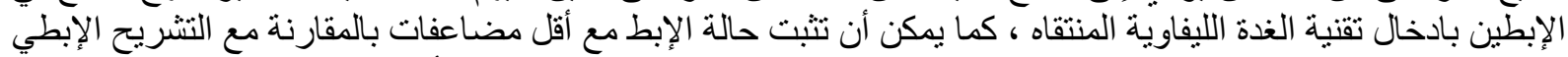

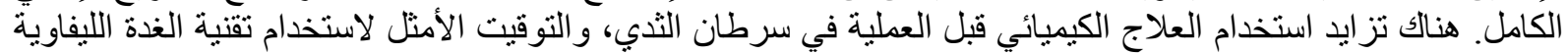

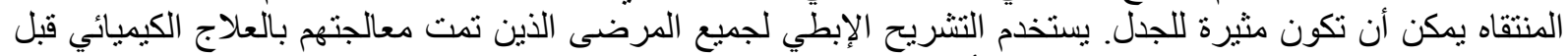

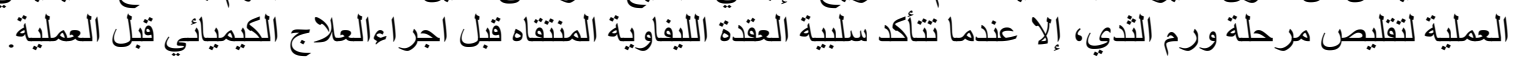

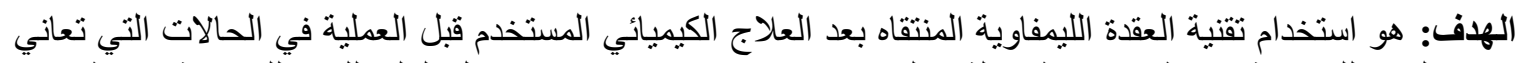

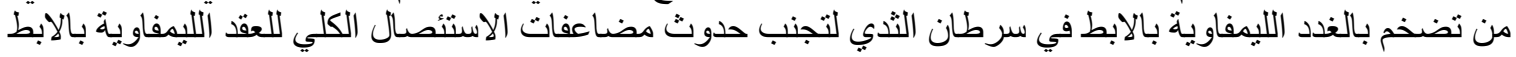

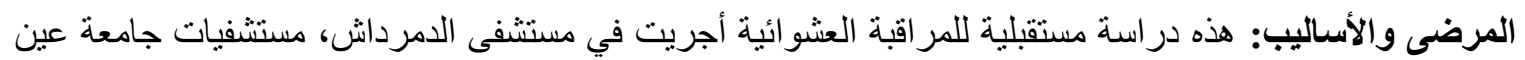

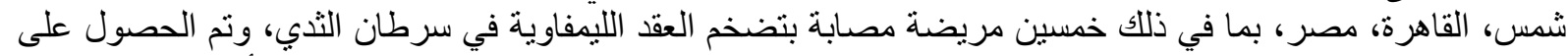

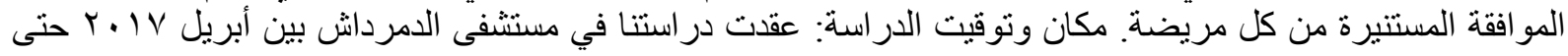

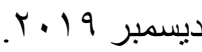

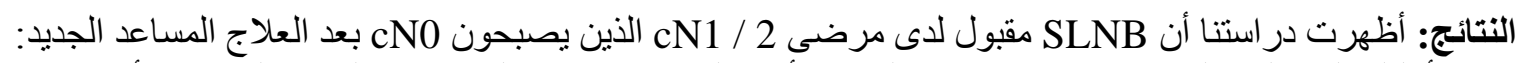

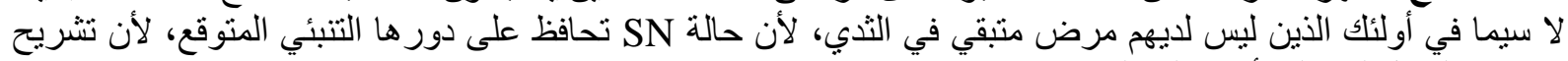

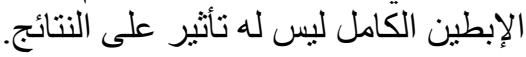

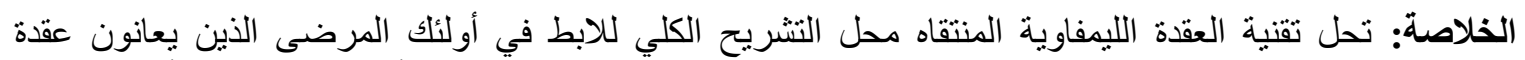

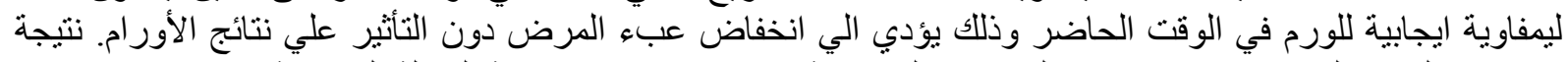

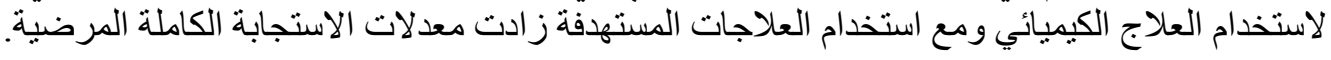

